\title{
Constrained Nonlinear Control Allocation with Singularity Avoidance using Sequential Quadratic Programming
}

\author{
Tor A. Johansen*, Thor I. Fossen* and Svein P. Berge**
}

\begin{abstract}
Control allocation problems can be formulated as optimization problems, where the objective is typically to minimize the use of control effort (or power) subject to actuator rate and position constraints, and other operational constraints. Here we consider the additional objective of singularity avoidance, which is essential to avoid loss of controllability in some applications, leading to a non-convex nonlinear program. We suggest a sequential quadratic programming approach, solving at each sample a convex quadratic program approximating the nonlinear program. The method is illustrated by simulated maneuvers for a marine vessel equipped with azimuth thrusters. The example indicates reduced power consumption and increased maneuverability as a consequence of the singularity-avoidance.
\end{abstract}

\section{INTRODUCTION}

A characteristic feature of control allocation problems is that there are more control signals available than the number of commanded forces and moments, such that the system is overactuated. Control allocation is used in many mechanical control systems in order to make a set of actuators jointly produce total forces and moments commanded by some higher level controller, and in addition optimize power consumption, take into account actuator constraints, and other objectives or constraints. Control allocation is used in flight control [1], [2], [3], [4], marine vessel control [5], [6], [7], [8], and other applications e.g. [9].

In the present work we focus on marine vessel control applications such as dynamic positioning and maneuvering. A marine vessel is usually equipped with a number of propulsion devices and control surfaces (generally called actuators), such as main propellers, rudders, fins, tunnel thrusters, and azimuth thrusters [10]. The scope of the paper is to present a solution for 3-DOF control allocation (horizontal plane motion), although the presented solution can also be used for 6-DOF motion. For horizontal plane motions (surge, sway and yaw) the control allocation problem is to select the control signals associated with the individual actuators in order to produce the commanded surge/sway forces and yaw moment (three generalized force components).

An actuator configuration is said to be singular if it cannot produce forces/moments in every direction in the 3-dimensional space of surge force, sway force and yaw moment. A singular configuration is characterized by the effect of every actuator being linearly dependent, which is also termed coplanar control, e.g. [11]. For a marine vessel equipped with some azimuth thrusters this may happen when the thrusters have equal azimuth angles. Since azimuth and rudder angle rates are often restricted

* Department of Engineering Cybernetics, Norwegian University of Science and Technology, NO-7491 Trondheim, Norway.

** MARINTEK, NO-7450 Trondheim, Norway.

Corresponding author: tor.arne.johansen@itk.ntnu.no to $1 \mathrm{deg} / \mathrm{s}$ or less, it takes several seconds to recover maneuverability after a near-singular actuator configuration. On the other hand, with a constant or slowly time-varying environmental disturbance it may be energy-optimal with singular operation as it is generally energy-optimal to align the orientation of rotatable thrusters with the direction of the disturbance force, if possible. It will be expensive or impossible to generate a force component orthogonal to this direction. Hence, there exists a tradeoff that should be explicitly addressed and one may want flexibility to tune the control allocation for energy-optimality versus maneuverability. The main contribution of this paper is that the cost function used for optimizing control allocation includes a term that penalizes the singularity of the configuration. This is important in dynamic positioning applications, but also in maneuvering applications that utilize azimuth thrusters or azipods, since the consequences of loss of maneuverability may be serious.

Most existing marine vessel control allocation approaches such as [6], [7] rely on a linear model that describes the relationship between the control signals and the generalized forces. ${ }^{1}$ This allows least-squares solutions to be found in an explicit form that allows the control allocation to be implemented using simple matrix computations such as generalized inverses. Such a linear model assumes that thruster azimuths or rudder angles are determined using another algorithm that takes into account power-optimality, non-singularity, constraints and other essential features, see [6], [12]. Various computational algorithms for constrained least squares control allocation, with emphasis on efficient real-time implementation without real-time optimization, are studied in [2], [3], [11], [12], [13], [14], [15]. Although explicit solutions are desirable due to their simple and efficient implementation, the use of real-time numerical optimization has been suggested, see [4], [8], [16], [17]. Its main advantage is that it admits considerable more flexibility in terms of handling nonlinearities and constraints, representing the power consumption more accurately than with a quadratic cost, dynamic reconfiguration, and is useful for optimizing rudder and azimuth angles.

The algorithm suggested here is also based on solving a constrained optimization problem numerically in real time, at each sampling instant. The objective is to minimize power consumption (or some other suitable objective) and at the same time avoid singular configurations and excessive rates of change of the azimuth angles. If the commanded forces cannot be attained, due to constraints on the attainable force, azimuth or rudder angles or their rates of change, the objective is shifted to minimize the error between the commanded and attained forces. The opti-

${ }^{1}$ Linear models are also common in flight control applications [2], [3]. 
mization formulation takes into account rate constraints on the azimuth/rudder angles, and possibly also rate constraints on the individual propeller control signals (such as pitch/speed). The present approach is therefore an extension of [4], [8], [16], [17], where least-squares and linear programming type of criteria are considered together with control amplitude and rate constraints, while non-singularity is not explicitly taken into account.

Closely related singularity avoidance objective functions have been suggested for spacecraft maneuvering control using control moment gyroscopes, see [18], [19], [20], [21] and the references therein, as well as in robotics, e.g. [22], [23]. However, they view the singularity avoidance problem either as one of running smoothly through singularities (at the cost of a transient torque error), or in the context of trajectory planning which is not natural in applications such as marine vessel dynamic positioning where the control problem is more one of disturbance rejection. Consequently, we take a quasi-dynamic control allocation approach, taking into account the actuator dynamics through rate constraints.

\section{3-DOF CONTROL ALLOCATION}

Consider a marine vessel equipped with $m$ force producing actuators (thrusters, rudders, propellers, etc.). The generalized force vector $\tau=\left(\tau_{X}, \tau_{Y}, \tau_{N}\right)^{T} \in \mathbb{R}^{3}$ produced jointly by the actuators is given by

$$
\tau=B(\alpha) u
$$

Its components are the surge force $\tau_{X}$, sway force $\tau_{Y}$ and yaw moment $\tau_{N}$. The vector $u \in \mathbb{R}^{m}$ contains the magnitude of the force produced by each individual actuator, uniquely related via invertible actuator characteristics to low-level control signals such as propeller speed or pitch, or intermediate-level control signals such as shaft torque or power [24]. The angle $\alpha_{i}$ is the azimuth of the $i$-th actuator, defining the direction of the force produced in the horizontal plane, and the vector $\alpha \in \mathbb{R}^{m}$ contains these angles. The $i$-th column of the $3 \times m$ matrix $B(\alpha)$ is given by

$$
B_{i}\left(\alpha_{i}\right)=\left(\begin{array}{c}
\cos \alpha_{i} \\
\sin \alpha_{i} \\
-\ell_{y i} \cos \alpha_{i}+\ell_{x i} \sin \alpha_{i}
\end{array}\right)
$$

The location of the $i$-th actuator in the horizontal plane is at $\left(\ell_{x i}, \ell_{y i}\right)$, in a coordinate system with origin in the center of rotation, positive $x$-axis forward, and positive $y$-axis towards starboard. The control allocation task is illustrated in Figure 1.

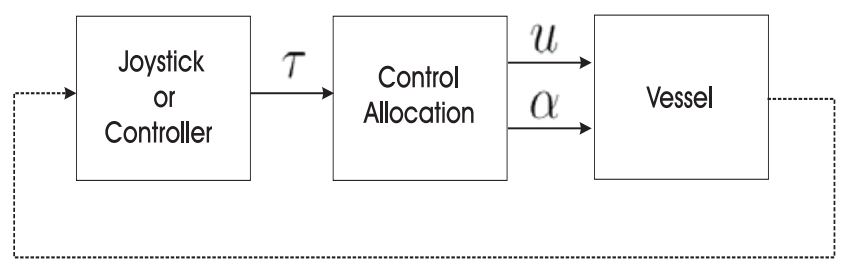

Fig. 1. Control allocation.

A singular configuration is characterized by $B(\alpha)$ not having full rank. This means that the attainable generalized force $\tau$ is restricted to a sub-space of $\mathbb{R}^{3}$ such that the system becomes under-actuated and controllability may be lost since the commanded generalized force vector $\tau$ cannot in general be attained. With controllability we mean the ability to produce a desired generalized force $\tau$ in any direction in $\mathbb{R}^{3}$ at every timeinstant, which is different from the common linear and nonlinear system-theoretic definitions of controllability. Near-singular actuator configurations have the same problem, as the control will easily saturate and the commanded generalized force cannot be attained. With three or more actuators $(m \geq 3)$ singularity usually only occurs for certain combinations of $\alpha_{1}, \ldots, \alpha_{m}$. Such a configuration must be avoided since it usually means that controllability will be lost for several seconds because the rate of change of the azimuths $\alpha$ may be small (typically $1 \mathrm{deg} / \mathrm{s}$ or less).

In order to make the control allocation robust and avoid nearsingular configurations, we require that the optimal $\alpha$ gives a feasible control allocation without a dramatic increase in power consumption also when $\tau$ is perturbed. This is achieved if $B(\alpha) B^{T}(\alpha)$ is not close to singular.

\section{QUASI-DYNAMIC OPTIMIZATION}

The following optimization problem is defined: Minimize with respect to $(\alpha, u, s)$ the criterion

$$
\begin{gathered}
J(\alpha, u, s)=\sum_{i=1}^{m} W_{i}\left(u_{i}\right)+s^{T} Q s+\left(\alpha-\alpha_{0}\right)^{T} \Omega\left(\alpha-\alpha_{0}\right) \\
+\frac{\varrho}{\varepsilon+\operatorname{det}\left(B(\alpha) B^{T}(\alpha)\right)}
\end{gathered}
$$

subject to

$$
\begin{aligned}
s & =\tau-B(\alpha) u \\
u_{\min } & \leq u \leq u_{\max } \\
\alpha_{\min } & \leq \alpha \leq \alpha_{\max } \\
\Delta \alpha_{\min } & \leq \alpha-\alpha_{0} \leq \Delta \alpha_{\max }
\end{aligned}
$$

The total power consumption is represented by the first term in the criterion, combining the power consumptions $W_{i}\left(u_{i}\right)$ of the individual actuators. The second term $s^{T} Q s$ penalizes the error $s$ between the commanded and achieved generalized force, cf. (4). This slack variable formulation is necessary in order to guarantee that the optimization problem always has a feasible solution. The diagonal weights in the matrix $Q>0$ are chosen so large that the constraint (4) is satisfied with $s \approx 0$ whenever possible. The maximum and minimum forces produced by the actuators are specified through the constraints (5), where $u_{\min }$ and $u_{\max }$ are vectors with lower and upper bounds for $u$, respectively. The azimuths, $\alpha$, are required to belong to given sectors defined by the vectors of lower and upper bounds, $\alpha_{\min }$ and $\alpha_{\max }$, cf. (6). Moreover, the rate-of-change in azimuths is constrained and minimized such that a large change is only allowed if this is necessary, represented by the third term in the criterion and the constraints (7). The matrix $\Omega>0$ is used to tune this objective, and the vector $\alpha_{0}$ contains the azimuths at the previous sample. Singularity is avoided through the fourth term in the criterion, where $\varepsilon>0$ is required to avoid numerical problems and $\varrho \geq 0$ is a weighting parameter. Essentially, a 
large $\varrho$ will lead to high maneuverability as the cost of increased steady-state power consumption. Conversely, a small $\varrho$ will give low power consumption under steady-state conditions at the cost of reduced maneuverability with highly dynamic commands.

Dynamic reconfigurability and fault handling can be achieved by dynamically changing the constraint limits or weighting matrices. For example, if actuator $i$ is not operative one may require $u_{i}^{\min }=u_{i}^{\max }=0$. Also, if an actuator can only produce force in a fixed direction, this can be represented as $\alpha_{i}=\alpha_{i}^{\min }=\alpha_{i}^{\max }=$ constant.

\section{LOCALLY CONVEX QUADRATIC PROGRAMMING REFORMULATION}

The above optimization problem is a nonlinear program that is generally non-convex and requires a significant amount of computations at each sample to solve. The first three terms in (3) are generally convex, while non-convexity is caused primarily by the non-linear constraint (4) and the fourth non-convex term in (3). This term was introduced to avoid singularity, i.e. designed to have peaks at singular configurations such that the optimization landscape contains "valleys due to the three first terms and "peaks due to the fourth term. ${ }^{2}$

For the purpose of implementing this control allocation strategy, a locally convex quadratic programming $(\mathrm{QP})$ reformulation is suggested. Since the azimuth vector $\alpha$ is required to change slowly from one sample to the next, a time-varying quadratic/linear approximation to the cost/constraints may be reasonable. The non-convex nonlinear program may therefore be replaced locally with a convex QP which can be solved efficiently and reliabily using standard numerical software, without prohibitive loss of performance. Recomputing some of the QP coefficients must be done at each sample, but this is fairly simple.

In order to get a convex quadratic approximation to the criterion, we approximate the first term (power consumption) by a quadratic term, and the last term (singularity avoidance) by a linear term by linearizing about the azimuth $\alpha_{0}$ and control $u_{0}$ from the previous sample optimal solution such that $\alpha=\alpha_{0}+\Delta \alpha$ and $u=u_{0}+\Delta u$ :

$$
\begin{aligned}
J_{Q P}(\Delta \alpha, \Delta u, s)= & \sum_{i=1}^{m}\left(\frac{d W_{i}}{d u_{i}}\left(u_{0, i}\right) \Delta u_{i}+\frac{d^{2} W_{i}}{d u_{i}^{2}}\left(u_{0, i}\right) \Delta u_{i}^{2}\right) \\
& +s^{T} Q s+\Delta \alpha^{T} \Omega \Delta \alpha \\
& +\frac{d}{d \alpha}\left(\frac{\varrho}{\varepsilon+\operatorname{det}\left(B(\alpha) B^{T}(\alpha)\right)}\right)_{\alpha=\alpha_{0}} \Delta \alpha
\end{aligned}
$$

Likewise, the constraints can be linearized as

$s+B\left(\alpha_{0}\right) \Delta u+\frac{\partial}{\partial \alpha}(B(\alpha) u) \mid \begin{aligned} & \alpha=\alpha_{0} \\ & u=u_{0}\end{aligned} \cdot \Delta \alpha=\tau-B\left(\alpha_{0}\right) u_{0}$

\footnotetext{
${ }^{2}$ This is similar to potential field obstacle avoidance in robotics, e.g. [23].
}

and

$$
\begin{aligned}
u_{\min }-u_{0} & \leq \Delta u \leq u_{\max }-u_{0} \\
\alpha_{\min }-\alpha_{0} & \leq \Delta \alpha \leq \alpha_{\max }-\alpha_{0} \\
\Delta \alpha_{\min } & \leq \Delta \alpha \leq \Delta \alpha_{\max }
\end{aligned}
$$

In the simulations below we have used the QLD public domain software, due to Schittkowski's implementation [25] based on Powell's original implementation [26] of the QP algorithm in [27]. The gradients above are computed numerically using finite differences. Convergence properties of QPs are well established [27], [28]. Convergence toward a local minimum of $J$ may happen. However, this is not regarded as a significant problem as the non-convex cost function typically has several global minima (due to symmetries) rather than sub-optimal local minima.
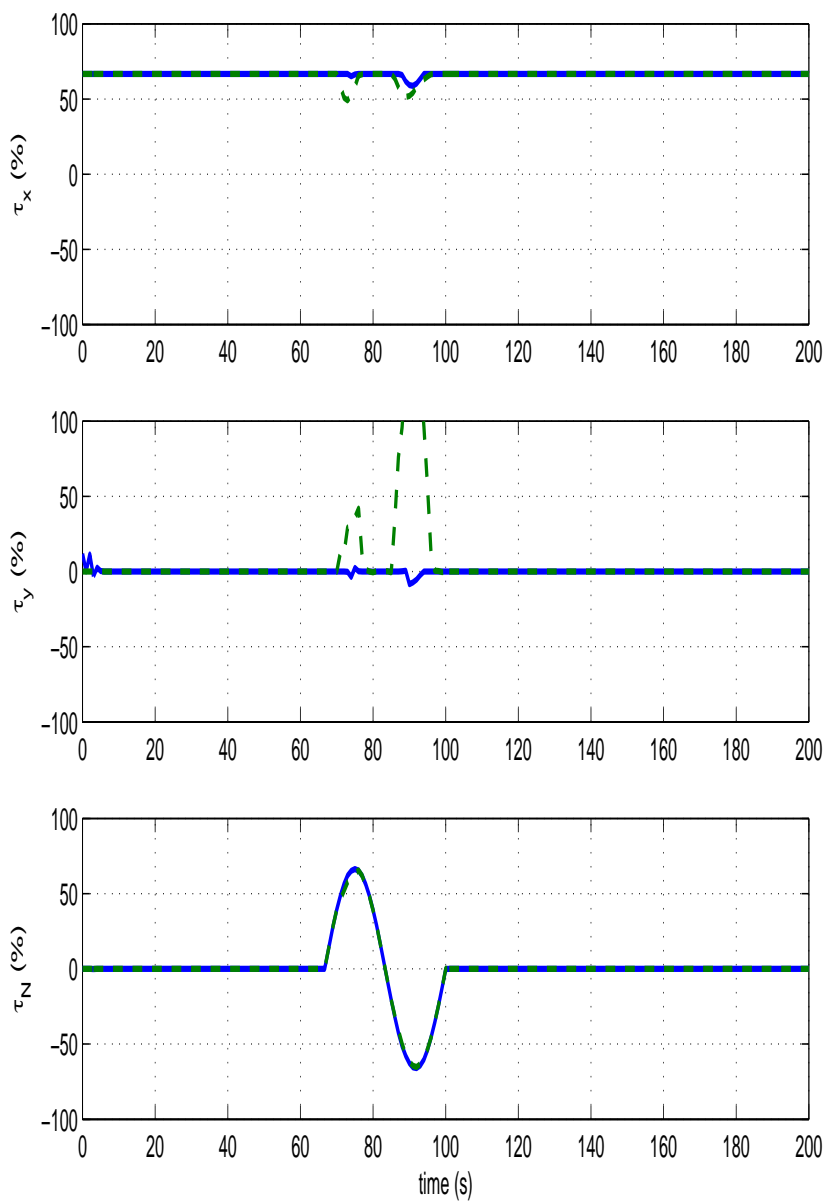

Fig. 2. Generalized forces (surge force $\tau_{X}$, sway force $\tau_{Y}$, and yaw-moment $\tau_{N}$ ) achieved by the thrust allocation algorithm. Solid curves are optimization with singularity avoidance, and dashed curves are optimization without singularity avoidance. Specifications are constant surge force, zero sway force, and a yaw-moment that are given by dashed-dotted curves that are almost indistinguishable from the one achieved using optimization with singularity avoidance.

\section{Simulation example}

Consider a marine surface vessel with four equal azimuth thrusters, one located at each "corner of the vessel, i.e. at locations $\left(\ell_{x}, \ell_{y}\right)=( \pm 20, \pm 5) \mathrm{m}$ relative to the center of rotation. 

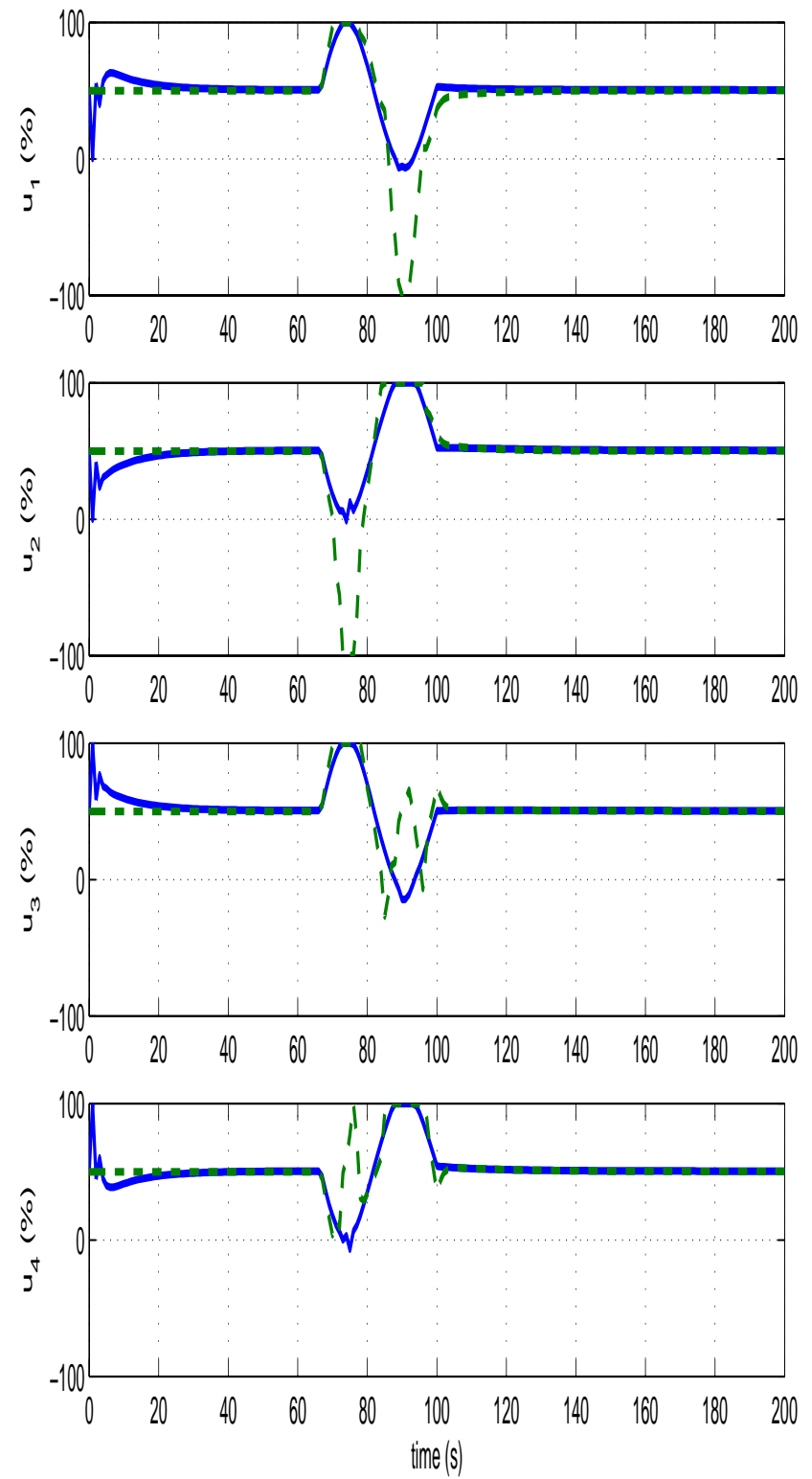

Fig. 3. Thrust provided by each thruster. Solid curves are optimization with singularity avoidance, and dashed curves are optimization without singularity avoidance.

We assume the azimuths are restricted to $\left|\alpha_{i}\right| \leq 25 \mathrm{deg}$, and $\left|\dot{\alpha}_{i}\right| \leq 1 \mathrm{deg} / \mathrm{s}$. The control signals are the thrusts provided by each thruster. Since the thrust is a quadratic function of propeller angular velocity and the power consumption is a cubic function of propeller angular velocity, [10], we take $W_{i}\left(u_{i}\right)=k_{i}\left|u_{i}\right|^{3 / 2}$. The simulation scenario assumes the vessel is operating at about $36 \%$ of total surge force, zero sway force, and then makes a heading change by commanding a time-varying yaw-moment of $30 \mathrm{~s}$ duration. Two different control allocation strategies have been simulated:

- The numerical optimization approach described above $(\varrho>$ $0)$.

- The same numerical optimization approach, but without sin-
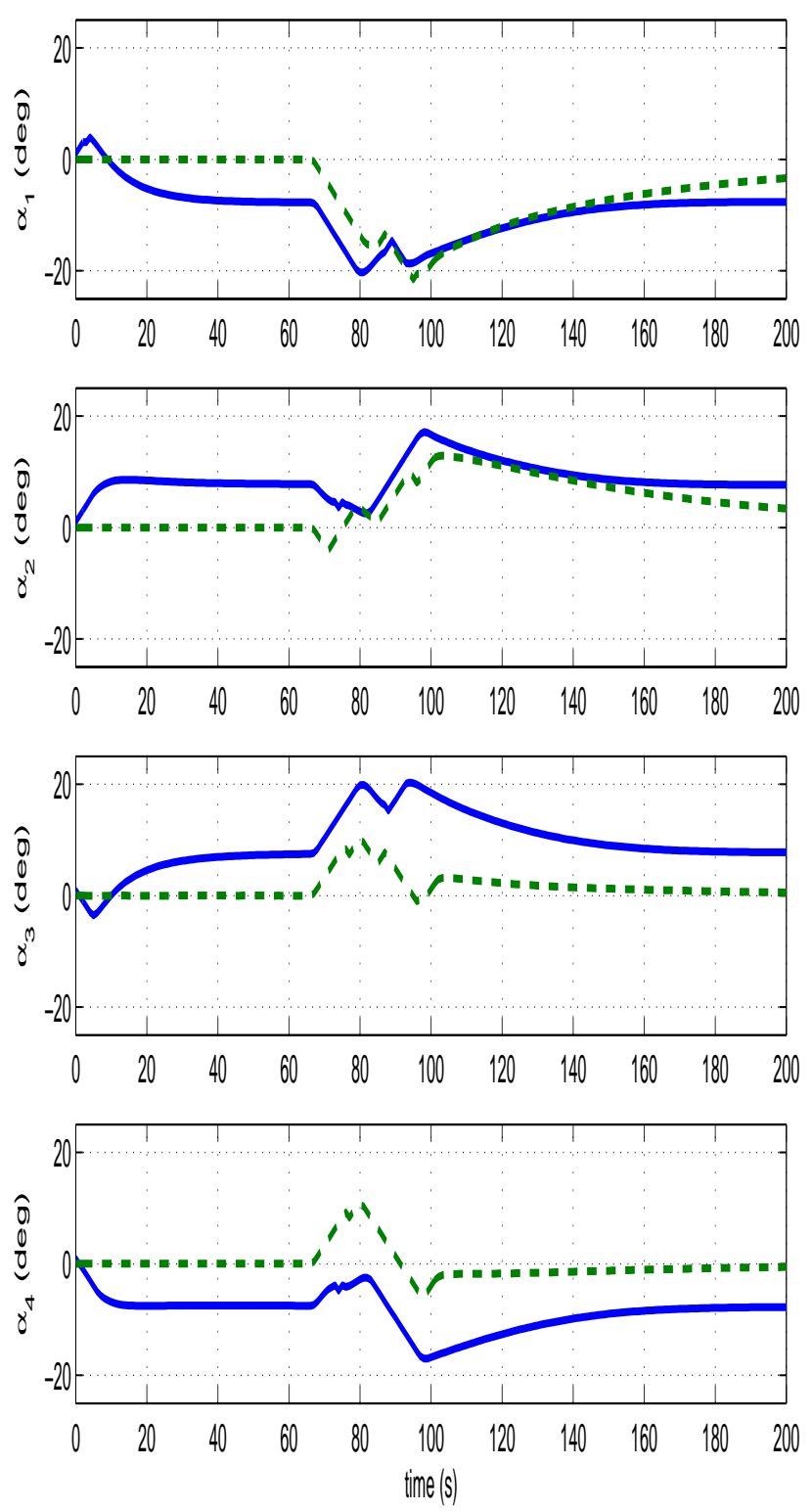

Fig. 4. Azimuth angles for each thruster. Solid curves are optimization with singularity avoidance, and dashed curves are optimization without singularity avoidance.

gularity avoidance $(\varrho=0)$.

Figures 2-5 show the achieved generalized forces, individual thrust for each thruster, individual azimuths for each thruster, and total power consumption (neglecting the relatively small power used to change the azimuths). It is observed that the optimization approach without singularity avoidance has lowest power consumption during steady-state operation (1-2\% less), as all thrusters produce forces almost parallel to the direction corresponding to a near-singular actuator configuration. However, during the course-changing maneuver, we observe that the approach with singularity-avoidance has dramatically less power consumption. Moreover, without singularity avoidance, the specified longitudinal and lateral thrusts deviate strongly 


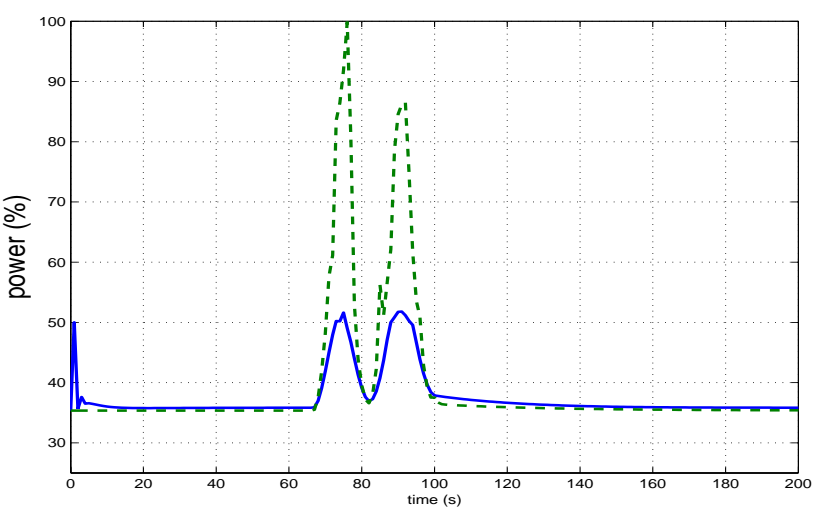

Fig. 5. Total power consumption. Solid curves are optimization with singularity avoidance, and dashed curves are optimization without singularity avoidance.

from the specifications because of partial loss of controllability due to the constraints during this maneuver. The example demonstrates the beneficial effect of singularity avoidance in terms of reduced power consumption and improved accuracy and reliability of the control allocation during maneuvering. The numerical computations per sample typically require $2 \mathrm{~ms}$ computation time on a $1 \mathrm{GHz}$ Pentium III.

\section{CONCLUSIONS}

It has been shown that singularities can be avoided in nonlinear constrained control allocation problems using a penalty term in a nonlinear programming formulation. The nonlinear program can be accurately approximated locally by a convex QP that admits efficient numerical solution in real-time using numerical QP software. Simulations indicate that power consumption and maneuverability can be significantly improved using the singularity avoidance penalty term. While the main disadvantage of the sequential quadratic programming approach lies in the complexity of the numerical software required to run in real time, the advantages include flexibility to allow nonquadratic cost functions, nonlinear models and multiple objective such as singularity avoidance.

\section{REFERENCES}

[1] D. Enns, "Control allocation approaches," in Proc. AIAA Guidance, Navigation and Control Conference and Exhibit, Boston MA, 1998, pp. 98-108.

[2] W. C. Durham, "Constrained control allocation," J. Guidance, Control and Dynamics, vol. 16, pp. 717-725, 1993.

[3] W. C. Durham, "Constrained control allocation: Three-moment problem," J. Guidance, Control and Dynamics, vol. 17, pp. 330-336, 1994.

[4] M. Bodson, "Evaluation of optimization methods for control allocation," J. Guidance, Control and Dynamics, vol. 25, pp. 703-711, 2002.

[5] N. A. Jenssen, Estimation and control in dynamic positioning of vessels, $\mathrm{Ph}$.D. thesis, Department of Engineering Cybernetics, Norwegian University of Science and Technology, 1981.

[6] O. J. Sørdalen, "Optimal thrust allocation for marine vessels," Control Engineering Practice, vol. 5, pp. 1223-1231, 1997.

[7] S. P. Berge and T. I. Fossen, "Robust control allocation of overactuated ships; experiments with a model ship," in Preprints IFAC Conference on Maneuvering and Control of Marine Craft, Brijuni, Croatia, 1997.

[8] I. Lindfors, "Thrust allocation methods for the dynamic positioning system," in Proc. 10th International Ship Control Symposium, Ottawa, Canada, 1993, pp. 3.93-3.106.

[9] W. Jackson, M. P. J. Fromherz, D. K. Biegelsen, J. Reisch, and D. Goldberg, "Constrained optimization based control of real time large-scale systems: Airjet object movement system," in Proc. IEEE Conf. Decision and Control, Orlando, 2001.
[10] T. I. Fossen, Marine Control Systems: Gudiance, Navigation and Control of Ships, Rigs and Underwater Vehicles, ISBN 82-92356-00-2, Marine Cybernetics, Trondheim, 2002.

[11] J. A. M. Petersen and M. Bodson, "Fast implementation of direct allocation with extension to coplanar controls," J. Guidance, Control and Dynamcis, vol. 25, pp. 464-473, 2002.

[12] K.-P. Lindegaard and T. I. Fossen, "Fuel efficient control allocation for surface vessels with active rudder usage: Experiments with a model ship," IEEE Trans. Control Systems Technology, accepted, 2003.

[13] K. A. Bordingnon and W. C. Durham, "Null-space augmented solutions to constrained control allocation problems," in Proc. AIAA Guidance, Navigation and Control Conference, Baltimore, MD, 1995, pp. 328-333.

[14] W. C. Durham, "Efficient, near-optimal control allocation," J. Guidance, Control and Dynamics, vol. 22, pp. 369-372, 1999.

[15] T. A. Johansen, "Reduced explicit constrained linear quadratic regulators," IEEE Trans. Automatic Control, vol. 48, pp. 823-828, 2003.

[16] W. C. Webster and J. Sousa, "Optimum allocation for multiple thrusters," in Proc. International Society of Offshore and Polar Engineers Conference (ISOPE-99), Brest, France, 1999.

[17] O. Härkegård, "Efficient active set algorithms for solving constrained least squares problems in aircraft control allocation," in Proc. IEEE Conf. Decision and Control, Las Vegas NV, 2002.

[18] D. E. Cornick, "Singularity avoidance control laws for single gimbal control moment gyros," in AIAA Conference paper 79-1698, 1979.

[19] B. R. Hoelscher and S. R. Vadali, "Optimal open-loop and feedback control using single gimbal control moment gyroscopes," J. Astronautical Sciences, vol. 42, pp. 189-206, 1994.

[20] S. R. Vadali and S. Krishnan, "Suboptimal command generation for control moment gyroscopes and feedback control of spacecraft," J. Guidance, Control and Dynamics, vol. 18, pp. 1350-1354, 1995.

[21] K. A. Ford and C. D. Hall, "Singular direction avoidance steering for control-moment gyros," J. Guidance, Control and Dynamics, vol. 23, pp. 648-656, 2000.

[22] Y. Nakamura and H. Hanafusa, "Inverse kinematic solutions with singularity robustness for robot manipulator control," J. Dynamic Systems, Measurement and Control, vol. 108, pp. 163-171, 1986.

[23] J. Borenstein and Y. Koren, "Real-time obstacle avoidance for fast mobile robots," IEEE Trans. Systems, Man, and Cybernetics, vol. 19, pp. 1179$1187,1989$.

[24] A. J. Sørensen and A. K. Ådnanes, "High performance thrust allocation scheme in positioning of ships based on power and torque control," in $D y$ namic Positioning Conference, Marine Technology Society, Houston TX, 1997.

[25] K. Schittkowski, "A FORTRAN code for quadratic programming," Tech. Rep., Mathematisches Institut, Universität Bayreuth, Germany, 1986.

[26] M. J. D. Powell, "ZQPCVX - a FORTRAN subroutine for convex programming," Tech. Rep. DAMTP/1983/NA17, University of Cambridge, UK, 1983.

[27] D. Goldfarb and A. Idnani, "A numerically stable dual method for solving strictly convex quadratic programs," Mathematical Programming, vol. 27, pp. 1-33, 1983.

[28] J. Nocedal and S. J. Wright, Numerical Optimization, Springer-Verlag, New York, 1999. 